\title{
Myasthenia Gravis - Exacerbation and Crisis
}

\section{(ㄷ) (1) (우) $\Theta$}

Authors

Michael Schroeter $^{1}$, Günther Thayssen ${ }^{2}$, Julia Kaiser ${ }^{3}$

Affiliations

1 Department of Neurology, University Hospital, University of Cologne, Cologne, Germany

2 Department of Neurology, University Hospital Hamburg-Eppendorf, Hamburg, Germany

3 Department of Neurology, LVR Clinic Bonn, Germany

Key words

myasthenia gravis therapy, review, myasthenia gravis crisis, intensive care unit

\author{
Bibliography \\ DOI https://doi.org/10.1055/s-0043-118441 \\ Neurology International Open 2018; 2: E10-E15 \\ (c) Georg Thieme Verlag KG Stuttgart · New York \\ ISSN 2511-1795 \\ Correspondence \\ Michael Schroeter MD \\ Dept. of Neurology \\ University Hospital, University of Cologne \\ 50924 Koeln \\ Germany \\ Michael.schroeter@uk-koeln.de
}

\begin{abstract}
Myasthenic exacerbation and crisis are most critical incidences in myasthenia gravis. Even nowadays myasthenic crisis is a life-threatening condition, with a lethality of $2-3 \%$. We review means of avoiding myasthenic exacerbation and crisis, elaborate on red flags and how to establish highly-active therapy in a timely manner. This includes the reasonable use of cholinesterase inhibitors, immunoadsorption or plasma exchange, as well as immunoglobulins and steroids. Immunosuppressive agents and monoclonal antibody therapy add to the therapeutic options.

Intensive care of myasthenic patients includes the management of dysphagia and delirium. Importantly, the perioperative management of patients undergoing thymectomy and weaning are specific challenges in the treatment of myasthenic patients in the ICU.

Establishing timely consequent immunosuppression and treatment of myasthenic patients in specialized outpatient centres help to avoid repetitive exacerbations and crises.
\end{abstract}

\section{Introduction}

With a total prevalence of myasthenia gravis (MG) of 15-20 per 100,000 , an estimated $15-20 \%$ of all myasthenia patients develop a myasthenic crisis in their course of the disease [1]. Frequently, crisis appears as an initial manifestation (20\%) or within the first year after disease onset. There is an individual recurrence risk of about $30 \%$ [2]. Lethality of 5-10\% has been reported [3].

These numbers are somewhat based on historical data and do not reflect current clinical reality, in which a manifest myasthenic crisis has become a rare event with good prognosis and low mortality (about 2-3\%)[4]. There are a number of reasons for this. Progress has been made in (i) establishing faster diagnosis, (ii) optimizing therapy management, in particular the consequent initiation of immunosuppressive therapy in the diagnosis of a generalized form, (iii) improving perioperative management, (iv) focussing the treatment of patients at specialized centers with a dedicated neurological intensive care unit, and ( $v$ ) by outreach efforts, e.g. the informational and education work of the German Myasthenia Society [5]. In this article we will summarize the state-of-the-art treatment of myasthenic crisis and exacerbation.

\section{Definition}

Myasthenic crisis is defined as a constellation of clinical conditions in which within a few days, or more rarely, a few hours, there is severe weakness of bulbar-innervated muscles or the respiratory muscles which restricts breathing or speech ability so severely that supportive feeding, intubation or ventilation are required [2]. Myasthenic crises thus require hospitalization in a monitored intermediate care facility or intensive care unit. Critical incidences are acute respiratory insufficiency, aspiration resulting in aspiration pneumonia, globus caused by restriction of the ability to swallow or cough, or generally the consequences and risks of immobility/ bed confinement and intensive care. 
- Table 1 Clinical red flags for exacerbation and crisis.

Febrile infection in the recent two weeks with of antibiosis Inverse aspiration: food and drink enter the nose when swallowing Insufficient swallowing: coughing or throat clearing after swallowing Insufficient cough impulse

Aphonic dysarthria: increasing phonation weakness in a sentence with hypernasal speech

Dropped head: head tips forward, fixed paresis of the head extensors

Dropped chin: lower jaw drops after (extended) chewing

New facial weakness

Vital capacity $<1500 \mathrm{ml}$ (men) / $<1000 \mathrm{ml}$ (women)

- Table 2 Medications which are frequently considered during the preliminary stage of myasthenic crises.

\begin{tabular}{|c|c|}
\hline Substance group & Examples \\
\hline Steriods, high-dose & Dexamethasone, triamcinolone (also locally) \\
\hline Gyrase inhibitors & Moxifloxacine \\
\hline Macrolides & $\begin{array}{l}\text { Azithromycine, clarithromycine, } \\
\text { telithromycine }\end{array}$ \\
\hline Lincomycins & Clindamycine \\
\hline Tetracyclines & Doxycycline \\
\hline $\begin{array}{l}\text { Non-nifedipine-type } \\
\text { calcium antagonists }\end{array}$ & Verapamile \\
\hline Antipsychotics & Opipramole, sulpiride \\
\hline
\end{tabular}

- Table 3 Red flags for a postoperative myasthenic crisis post-thymectomy $[2,11]$.

\begin{tabular}{|l|l|}
\hline Parameter & Critical value \\
\hline Vital capacity & $<2-2.9 \mathrm{l}$ \\
\hline Long-term myasthenia & $>6$ years \\
\hline Pyridostigmine daily dose & $>750 \mathrm{mg} / \mathrm{d}$ \\
\hline History of chronic pulmonary disease & Yes \\
\hline Preoperative bulbar symptoms & Present \\
\hline History of myasthenic crisis & Yes \\
\hline Intraoperative blood loss & $>1000 \mathrm{ml}$ \\
\hline High AChR-Ab titer & $>100 \mathrm{nmol} / \mathrm{l}$ \\
\hline Pronounced decrement at $3 \mathrm{~Hz}$ stimulation & $>18 \%$ \\
\hline
\end{tabular}

The concept "exacerbation of a myasthenic syndrome" has long been arbitrarily defined [6, 7]. However, the definition has been gained considerable practical importance in Germany since decision of the German Joint Federal Committee (G-BA) stating that the administration of polyvalent immunoglobulins is indicated during exacerbations[7]. Based on clinical experience and relevant study protocols, the following operational criteria for exacerbation can be formulated: (i) Temporal criterion: a progressive deterioration of the myasthenic syndrome can be observed in recent days, and no longer than 30 days.

(ii) Subjective criterion: a restriction of bulbo-pharyngeal functions, head-neck muscles or extremity strength affecting everyday life, or beginning breathing weakness with reduced cough impulse.

(iii) Objective criterion: the qualitative myasthenia gravis score (QMG, [9]) is at least 8 points, with no more than 5 points resulting from the ocular findings. The QMG refers to the clinical condition without an effect of cholinesterase inhibitors.

Exacerbation should thus be presumed if all three criteria have been met.

Generally, exacerbation requires inpatient treatment until there is a distinct improvement in clinical symptoms, measures to secure the air passages are no longer required and oral feeding is possible without risk of aspiration.

\section{Clinical Signs of Exacerbation or Imminent Crisis}

Exacerbation of myasthenia must always be regarded as a possible prodromal stage of a crisis and requires rapid and consequent treatment. There is a substantial risk of acute worsening with the need for intensive care, particularly during initial manifestation or with concomitant bacterial or viral infection, as well as resulting from halfhearted or discontinued immunosuppression. - Table 1 summarizes clinically ominous signs of an imminent crisis (red flags).

Furthermore, certain medications play an important role in worsening myasthenia. - Table $\mathbf{2}$ highlights drugs that are particularly critical with respect to crisis. Comprehensive lists are available on the internet. However these lists are of overall low evidence, collecting descriptions of case reports and personal experiences [13].

\section{Exacerbation and crisis after thymectomy}

Thymectomy as myasthenia therapy should never be done as an emergency measure but should be performed only on stabilized patients. Exacerbation and crisis after thymectomy are particular risks of thymectomy, especially when the compensation of myasthenic symptoms is fragile already before surgery [10].

Although thymectomy in patients with acetylcholine receptor antibody (AChR-Ab) positive generalized myasthenia is undisputed, the procedure is not risk-free. Frequently a temporary postoperative deterioration of myasthenia is observed, and the positive effects will manifest only months or years later. A stable condition before surgery is the decisive predictor for a postoperative uncomplicated course of the illness. - Table 3 summarizes predictors for an unfavourable course with the need for prolonged ventilation. It is interesting to note that these data from retrospective studies suggest that medication with cortisone and immunosuppressants does not predict a complicated course. This is consistent with the clinical experience that a consistent and clinically effective anti-myasthenic therapy is more important for an uncomplicated course after thymectomy than low daily doses of cortisone or the avoidance of immunosuppressive therapy, e.g., using azathioprine $(\triangleright$ Table 4). 


\section{Perioperative management}

Typically, the pyridostigmine dose should be low or moderate $(<360 \mathrm{mg} / \mathrm{d})$ before thymectomy.

In that case it is generally sufficient that peri-operatively the pyridostigmine dose is continued orally or via nasogastric tube without interruption. If there is a need for parenteral continuation, it is possible via perfusor. The parenteral daily dose corresponds to a thirtieth of the oral dose, thus $10 \mathrm{mg} / 24 \mathrm{~h}$ is the parenteral equivalent of the oral $300 \mathrm{mg}$ daily dose. Neostigmine is available as an alternative for parenteral administration of pyridostigmine; the equivalent dose is about one eightieth; that is, $3.6 \mathrm{mg} / 24 \mathrm{~h}$ of neostigmine is clinically equivalent to a daily oral dose of $300 \mathrm{mg}$ of pyridostigmine.

Anaesthesia should be performed by an anaesthesiologist experienced with myasthenia. Although the special anaesthesiological issues relating to myasthenia patients cannot be the subject of this overview, certain principles should be kept in mind [12]:

(i) the use of low-dose benzodiazepines during premedication

(ii) the avoidance of non-depolarizing muscle relaxants (depolarizing relaxants are absolutely contraindicated)

(iii) in favour of deepened inhalation anaesthesia

(iv) the selection of rapidly or ultra-rapidly effective narcotics and analgesics, and

(v) extended postoperative monitoring in the intensive care unit.

If cortisone therapy is maintained above the Cushing threshold

for an extended period of time, a perioperative Addison's substitution should be performed due to an insufficiency of endogenous cortisone production.

\section{Treating Myasthenic Crisis}

Multi-dimensional treatment of myasthenic crisis include symptomatic pyridostigmine therapy, causal acute therapy, initiation or modification of long-term immunosuppressive therapy as well as management of clinical symptoms. For the latter we would like to focus on weaning from the respirator as well as swallowing assessment / dysphagia therapy.

\section{Symptomatic therapy}

If the patient has been intubated and ventilated, there is basically no need for administering pyridostigmine. Instead, the situation can be used for a "drug holiday" since high doses of pyridostigmine are frequently used in the prodromal phase of the crisis. Increased bronchial mucus secretion by cholinesterase inhibitor increases the rate of pulmonary complications and is another reason to discontinue cholinesterase inhibitors when ventilation is needed. However, the parenteral use of pyridostigmine or neostigmine in moderate doses is an important but ultimately auxiliary therapeutic measure for weaning from the respirator.

\section{Plasma exchange/immunoadsorption}

Plasma exchange and immunoadsorption (PLEX/IA) are first-choice methods for treating a myasthenic crisis $[13,14]$. There are retrospective and comparative studies of both of these approaches. At the end, PLEX is more commonly used in UK and U.S. but IA more frequently used in continental Europe countries for historic, approval and economic reasons. Advantages of immunoadsorption
- Table 4 Latency of some myasthenia therapies "Dip" is a transient clinical deterioration at the start of therapy.

\begin{tabular}{|l|l|}
\hline Therapy & \multicolumn{1}{|c}{ Latency } \\
\hline PLEX/IA & Few days \\
\hline IVIG & Few days (dip possible?) \\
\hline Cortisone & $3-4$ weeks (“dip” frequent!) \\
\hline Azathioprine & $6-12$ months \\
\hline Mycophenolate & $6-12$ months (???) \\
\hline Rituximab & $2-3$ months \\
\hline Thymectomy & Months - years \\
\hline
\end{tabular}

versus plasma separation are lower circulatory load and elimination of the need for donor plasma or human albumin. Both methods are complex and not always available, and have the common risk of large-lumen central venous access, which, however, can be avoided in some patients with immunoadsorption. Treatment with 5-6 sessions has been shown to be effective. An effect will be apparent within a few days. The effect of PLEX/IA can be documented by the titer decrease of myasthenia-specific antibodies.

\section{Immunoglobulins}

Immunoglobulins (IVIG) are likewise a first-choice response to a myasthenic crisis. Comparative studies of PLEX/IA and IVIG have produced comparable results in both treatment groups, although in individual cases a delayed effect of IVIG (up to 2 weeks) or even an initial deterioration ("dip" similar to the initiation phase of cortisone therapy) has been reported. The typical IVIG dose is $0.4 \mathrm{~g} /$ $\mathrm{kg}$ body weight (bw), on each of five successive days $[7,13,15]$.

Clinical experience has often shown, contrary to the abovenamed studies, a more pronounced and rapid onset of the effects of PLEX/IA compared to IVIG. The administration of IVIG followed by subsequently PLEX/IA is occasionally clinically practiced, but pharmacodynamical considerations challenge this concept.

\section{Cortisone}

Cortisone is not a first-line response to a myasthenic crisis. Nevertheless, administration should be started parallel to IVIG or PLEX/ IA, since a positive effect can be expected 3-4 weeks afterwards, that is, when, on the one hand, the effect of PLEX/IA or IVIG starts to decline, and, on the other hand, a new regime of immunosuppression will not be sufficiently clinically effective yet. The initial dose is e. g., $100 \mathrm{mg}$ of methylprednisolone administered orally or intravenously. If the patient is already artificially ventilated cortisone can be started without dose inclination.

\section{Immunosuppressants}

Immunosuppressant therapy is always indicated to start in patients with myasthenic crisis. The current German Neurological Society (DGN) guideline provides support in this regard [13]. The sole immunosuppressive drug approved for myasthenia is azathioprine. The guideline identifies several alternatives in case of intolerance; In Germany, mycophenolate is primarily recommended by the g-Ba 
[8]. Occurrence of myasthenic crisis despite ongoing and effective immunosuppressive therapy should prompt therapy escalation. The use of rituximab has proven to be effective in treating both AChR-Ab and MuSK-Ab-positive myasthenia $[13,16]$. Rituximab is administered twice at a 14-day interval, in a fixed $1 \mathrm{~g}$ dose analogously to its application in rheumatic diseases. This cycle is repeated after one year, or after CD19-positive cells have re-occurred in the measurable range. Clinical effects of rituximab can be expected after 8-12 weeks. Rituximab therapy can start directly after completing of PLEX/IA or IVIG cycles [17]. Utilization of other B-cell-directed antibodies such as bortezomib or ocrelizumab is pathophysiologically justified; however, only experimental and little clinical experience has been reported. The antibody eculizumab directed against the terminal membrane-attacking complex of the complement system promises a new, applicable add-on therapeutic principle recently approved for severe therapy-refractory AChR-Ab positive myasthenia. It may be an alternative in selected patients to the repetitive administration of IVIC in patients with prolonged ICU stays and failure to respond sustained and adequately to the above mentioned therapies.

\section{Exacerbation}

Administration of immunoglobulins is the first choice of treatment for exacerbation. The dosage is $0.4 \mathrm{~g} / \mathrm{kg}$ of body weight five days in a row or $1 \mathrm{~g} / \mathrm{kg}$ of body weight divided among 2(-3) days. Parallel to the recommendation for myasthenic crisis, cortisone will be an important therapeutic option. During an unstable situation, however, a gradual incline of cortisone is preferred to avoid a cortisone dip. Due to IVIG administration, the delayed effectiveness is clinically irrelevant; thus these therapies should be implemented in a complementary way.

Exacerbation should also be an incentive to initiate immunosuppressive therapy or to critically evaluate an existing therapy, including patients' adherence. Effective immunosuppression by azathioprine can only be assumed if an absolute lymphocyte count of $0.6-$ $1.0 / \mathrm{nl}$ has been verified, with no leukopenia below 3.0/nl. If this is not the case, stabilization can be attempted by dose optimization. Otherwise, escalation should be considered, e. g., to rituximab therapy.

\section{Management of the Myasthenic Crisis in the Neurological Intensive Care Unit}

Management of a myasthenic crisis should be carried out in a neurological intensive care unit with a physician and nursing team with relevant experience in myasthenia. In the following, we will focus on three essential aspects of the management of a myasthenic crisis patient in the intensive care unit: breathing and ventilation, swallowing assessment and training as well as delirium.

\section{Breathing and ventilation}

There must be clinical indication for ventilation. In addition to static indications ( $\triangleright$ Table 5), dynamics of the decrease in vital capacity appear to be an appropriate paraclinical parameter for the selection of the proper time point for an early elective intubation. In addition to ventilation-related parameters, determination of a
- Table 5 Parameters for bedside ventilation during myasthenic crisis [18].

\begin{tabular}{|l|c|c|c|c|}
$\begin{array}{l}\text { Criterion/ } \\
\text { indication }\end{array}$ & Normal & Intubation & Weaning & Extubation \\
\hline $\begin{array}{l}\text { Vital capacity } \\
(\mathrm{ml} / \mathrm{kg} \mathrm{BW})\end{array}$ & $>60$ & $<20$ & $>15$ & $>25$ \\
\hline $\begin{array}{l}\text { Negative airway } \\
\text { pressure }\left(\mathrm{cm} \mathrm{H} \mathrm{H}_{2} \mathrm{O}\right)\end{array}$ & $>70$ & $<30$ & $>20$ & $>40$ \\
\hline $\begin{array}{l}\text { Positive airway } \\
\text { pressure }\left(\mathrm{cm} \mathrm{H} \mathrm{H}_{2} \mathrm{O}\right)\end{array}$ & $>100$ & $<40$ & $>40$ & $>50$ \\
\hline
\end{tabular}

higher degree of swallowing inhibition and (silent) aspiration may also prompt intubation.

Cholinesterase inhibitor should be paused after intubation (see above). Typically pressure-regulated breathing modes are selected; emphasis is placed on respiratory toilet measures, possibly via bronchoscopy, appropriate pneumonia prophylaxis and physical measures due to the limited coughing force.

During the weaning phase, pressure-regulated ventilation is interrupted by intervals of spontaneous breathing with continuous positive airway pressure (CPAP) in a structured manner with extending intervals CPAP and an un-assisted spontaneous breathing in further steps. Causal therapy of the crisis (PLEX/IA or IVIG) should be regarded adequately effective as a prerequisite to consider extubation. In addition, satisfactory ventilation-related parameters (see $>$ Table 5), sufficient cough impulse and adequate ability to swallow are expected. As with other neuromuscular diseases, an early elective tracheostomy is indicated if crisis is prolonged. Non-invasive ventilation are discouraged during myasthenic crisis, not least because of the risk of aspiration when dysphagia is also present [18]. In the case of aspiration pneumonia, early antibiosis should be initiated after taking material for microbiological diagnostics; as antibiotics cephalosporins are preferred.

\section{Dysphagia and treatment options in the ICU}

Dysphagia is common among patients with a bulbar disorder, especially among those who are MuSK-positive. In MuSK-positive patients, dysarthria is often the initial symptom, but in these cases, dysphagia and weakness of the masticatory muscles are usually present. The incidence of dysphagia in all forms of myasthenia is reported as $17 \%$ for the initial symptom and $53 \%$ in the subsequent course of the disease $[1,19]$.

In any case, dysphagia increases the risk of complications; in particular, there is an additional risk of pneumonia. Oropharyngeal symptomatology, often discrete, leads to the diagnosis of generalized myasthenia. When using the Besinger score (or modified as QMG), chewing and swallowing symptoms are also evaluated in several degrees of severity.

Severe dysphagia is always an indication for intensive medical surveillance of the patient with the possibility of intubation and invasive ventilation due to the risk of aspiration. In the case of less severe but longer-lasting dysphagia, weight loss is frequently a prominent symptom, which is why the patient's weight should al- 
ways be determined in the course of long-term care of myasthenia patients. The differential diagnosis of myasthenia should always be included in evaluating the cause of dysphagia. With regard to myasthenia persistent dysphagia is an indication for the initiation or escalation of drug treatment. If so indicated in severe cases, nutrition can also be secured by nasalgastral tube until therapy is successful. Likewise, in the context of intensive medical treatment, additional therapeutic measures are often helpful such as adaptation of food, since often solids or even watery fluids cannot be swallowed. Thickened liquid or mushy food can help in this regard.

In the intensive care unit, FEES examination ("flexible endoscopic evaluation of swallowing") adds valuable information to the assessment of dysphagia. It identifies silent aspiration, and may support the indication for intubation complimentary to clinical symptoms. Likewise it is useful to establish individual therapeutic procedures in collaboration with speech therapists, since different observable symptoms correlating with certain pathophysiological manifestations require different therapeutic treatment strategies $[20,21]$. Likewise, the dietary regimen of the intensive care patient should be coordinated among the multidisciplinary team and adapted in the course of medical treatment.

\section{Delirium}

Delirium is a common problem in neurological intensive care units. Patients in myasthenic crisis develop markedly hypermotoric delirious states; however, exact incidences are not known. Whether specific pathophysiological aspects related to myasthenia are involved, e. g., dysregulation of the cholinergic system after discontinuation and re-introduction of cholinesterase inhibitors, is speculative. Based on our clinical experience, a high dose of pyridostigmine, prolonged ventilation, the delayed use of PLEX/IA, and high-dose administration of cortisone characterize a typical situation in which delirium occur.

Delirious and anxious-agitated patients who have not yet been or are no longer intubated usually respond well to lorazepam. In this situation, respiratory suppression plays a less important role than agitation and the muscular prostation. The occurrence of delirium in pre-crisis situation, however, is always an ominous sign of an impending need for ventilation within the next few hours.

In the weaning phase, delirium makes it difficult to assess cough impulse, breathing and swallowing, and prevents reliable communication with the patient. In this phase, the delirium limits clinical progress, extends the ventilation time, and stay in the intensive care unit. Although benzodiazepines and phenothiazine antipsychotics can be used for treatment, they should be administered in the smallest possible doses as they pose the risk of medication-induced myasthenia. In this regard haloperidol may be preferred, but parenteral use is not advised. Some low-potency neuroleptics such as pipamperone and melperone have proven useful in this situation, but are also only enterally available. Experience with dexmedetomidine in myasthenia patients is still not sufficient to recommend this therapy. All in all, treatment of delirium in patients in myasthenic crisis is difficult and often lengthy, requiring an individualized treatment regimen. Early initiation of day-structuring activities, establishment of a day-night rhythm, non-drug measures, verticalization and mobilization, physio- and ergotherapy as well as initiation of rehabilitation activities help to overcome this prob- lem. Clinical improvement of the myasthenic symptoms by means of rapid introduction of consistent causal therapy is a prerequisite for a favourable course.

\section{Summary for Clinical Care}

Cardinal symptoms of a myasthenic crisis are signs of muscular weakness of the respiratory and oro-pharyngeal musculature, which can lead to rapid decompensation with respiratory failure and aspiration due to disease-specific muscular prostration within minutes. Crises occur particularly in the initial phase of severe generalized myasthenia and are often triggered by errors in treatment or medication side effects. Exacerbation, a progressive deterioration of the symptoms which lead to a functionally relevant impairment of the bulbo-pharyngeal musculature should be addressed in a timely manner.

Myasthenia patients with functional deficits of the respiratory and swallowing function require intensive medical supervision. An attempt should be made to avoid respiratory decompensation by administering doses of 1.0 to $2.0 \mathrm{mg}$ of pyridostigmine. In the case of bulbar insufficiency with a specific risk of aspiration, however, the airways must be secured at an early stage by intubation. To avoid cholinergic side effects, pyridostigmine treatment should only be performed in a lower dose range.

Plasmapheresis or equivalent immune adsorption can influence the course of the disease very favourably within a few days. By using these early in the case of exacerbation which does not respond sufficiently to pyridostigmine, intubation and ventilation can be frequently avoided. As an alternative to PLEX/IA, intravenous immunoglobulin therapy can be performed in the case of e. g., manifest infection.

In the case of exacerbation with significant bulbar involvement, corticoids are not indicated due to their delayed effect and regular worsening during the first weeks of treatment. After stabilization, consistent immunosuppression with corticoids and azathioprine should be initiated during the further progression of the disease.

In the case of aspiration pneumonia, early antibiosis should be initiated after asservation matreials for microbioogical diagnostics; cephalosporins are preferred.

\section{Conflict of interest}

Since 2014, M. Schroeter has received speaking fees from Alexion, Biogen, Genzyme/Sanofi, Grifols, Miltenyi Biotec, Novartis. No conflict of interest has been declared by G. Thayssen and J. Kaiser.

References

[1] Meriggioli MN, Sanders DB. Autoimmune myasthenia gravis: Emerging clinical and biological heterogeneity. Lancet Neurol 2009; 8: 475-490

[2] Lacomis D. Myasthenic crisis. Neurocrit Care 2005; 3: 189-194

[3] Alshekhlee A, Miles JD, Katirji B et al. Incidence and mortality rates of myasthenia gravis and myasthenic crisis in US hospitals. Neurology 2009; 72: 1548-1554 
[4] Thomas CE, Mayer SA, Gungor Y et al. Myasthenic crisis: clinical features, mortality, complications, and risk factors for prolonged intubation. Neurology 1997; 48: 1253-1260

[5] Bungard S, Rohn H. Döbler: Zertifizierung von Myasthenie-Zentren Entwicklung und Umsetzung eines Zertifizierungsverfahrens für Patientenorganisationen. Z Eval Fortb Qual Gesundhwes 2011; 105 : 49-53

[6] Zinman L, $\mathrm{Ng} \mathrm{E}$, Bril V. IV immunoglobulin in patients with myasthenia gravis: A randomized controlled trial. Neurology 2007; 68: 837-841

[7] Alabdali M, Barnett C, Katzberg H et al. Intravenous immunoglobulin as treatment for myasthenia gravis: Current evidence and outcomes. Expert Rev Clin Immunol 2014; 10: 1659-1665

[8] Anonym Bundesausschuss AG. Verordnungsfähigkeit von zugelassenen Arzneimitteln in nicht zugelassenen Anwendungsgebieten (off-label use). BAnz AT 10.07.2014 B4

[9] Bedlack RS, Simel DL, Bosworth $\mathrm{H}$ et al. Quantitative myasthenia gravis score: assessment of responsiveness and longitudinal validity. Neurology 2005; 64: 1968-1970

[10] Jamal BT, Herb K. Perioperative management of patients with myasthenia gravis: Prevention, recognition, and treatment. Oral Surg Oral Med Oral Pathol Oral Radiol Endod 2009; 107: 612-615

[11] Watanabe A, Watanabe T, Obama T et al. Prognostic factors for myasthenic crisis after transsternal thymectomy in patients with myasthenia gravis. J Thorac Cardiovasc Surg 2004; 127: 868-876

[12] Gritti P, Sgarzi M, Carrara B et al. A standardized protocol for the perioperative management of myasthenia gravis patients. Experience with 110 patients. Acta Anaesthesiol Scand 2012; 56: 66-75

[13] Wiendl H. (federführend). Diagnostik und Therapie der Myasthenia gravis und des Lambert-Eaton-Syndroms. https://www.dgn.org/ leitlinien/3005-II-68-II-diagnostik-und-therapie-der-myastheniagravis-und-des-lambert-eaton-syndroms. Last access 22.07.2017
[14] Loschiavo C, Grecò M, Polo A et al. The use of therapeutic apheresis in neurological diseases and comparison between plasma exchange and immunoadsorption. G Ital Nefrol 2015; 32: pii: gin/32.1.10

[15] Stangel M, Baumann U, Borte M et al. Treatment of neurological autoimmune diseases with immunoglobulins: First insights from the prospective SIGNS registry. J Clin Immunol 2013; 33 (Suppl 1): S67-S71

[16] Iorio R, Damato V, Alboini PE et al. Efficacy and safety of rituximab for myasthenia gravis: A systematic review and meta-analysis. J Neurol 2015; 262: 1115-1119

[17] Melzer N, Ruck T, Fuhr P et al. Clinical features, pathogenesis, and treatment of myasthenia gravis: A supplement to the Guidelines of the German Neurological Society. J Neurol 2016; 263: 1473-1494

[18] Rabinstein AA. Noninvasive ventilation for neuromuscular respiratory failure: when to use and when to avoid. Curr Opin Crit Care 2016; 22: 94-91

[19] Godoy DA, Mello L], Masotti L et al. The myasthenic patient in crisis: An update of the management in Neurointensive Care Unit. Arq Neuropsiquiatr 2013; 71: 627-639

[20] Higo R, Nito T, Tayama N. Videofluoroscopic assessment of swallowing function in patients with myasthenia gravis. J Neurol Sci 2005; 231 : 45-48

[21] Dziewas R, Glahn ], Helfer C et al. Flexible endoscopic evaluation of swallowing (FEES) for neurogenic dysphagia: training curriculum of the German Society of Neurology and the German stroke society. BMC Med Educ 2016; 16: 70 